\title{
Muddy waters
}

\author{
Elizabeth Loder head of research
}

The BMJ

Radiologists, observes Giles Maskell (doi:10.1136/bmj.k3754), are ambivalent about incidental findings. They know that some patients are harmed by the diagnostic procedures or treatments that may ensue. But others benefit from detection of unsuspected conditions. He illustrates the problem with an unsettling tale. Two fictional patients undergo magnetic resonance imaging for back pain. One dies from complications of a workup for an incidentaloma determined to be harmless. The other dies from an incidentaloma that was missed: cancer that in hindsight was visible in its early stages on the MRI. "These are muddy waters," writes Maskell. He suggests that while an ethicist might be able to determine whether overdiagnosis or underdiagnosis caused the greater wrong, "having witnessed variants of both scenarios, I feel equally distraught in each case."

It isn't easy to identify overdiagnosis in the context of screening, say John Brodersen and colleagues (doi:10.1136/bmj.k3494). It's even more difficult to explain the problem to patients and the public. Overdiagnosis can be detected at the population level by large, long term follow-up studies of screening trials. It's much harder to identify in an individual patient. To muddy the waters further, the harmful effects of overdiagnosis often appear to be benefits. Patients with indolent disease seem cured, but they didn't need treatment in the first place. These apparent good outcomes inflate survival estimates and make screening seem better and more desirable than it is, a phenomenon termed the "popularity paradox." There are no easy answers, say the editorialists. But better prognostic methods and tools to identify overdiagnosis in individuals are top priorities.

Contradictions also abound in this week's research offerings. Diclofenac is a commonly used nonsteroidal anti-inflammatory drug that is effective for many types of pain. But a large Danish study shows the benefit comes at the price of an increased risk of stroke and cardiac problems in patients who initiate treatment (doi:10.1136/bmj.k3426). The risk is highest in comparison with similar patients who do not start an NSAID but is also elevated in comparison with patients who begin ibuprofen or naproxen.

Another study this week shows that statins, too, are a mixed bag (doi:10.1136/bmj.k3359). When used for primary prevention of cardiovascular events in old or very old adults, statins were beneficial only in those aged 75 to 84 who also had diabetes. Even this advantage waned after age 85 and disappeared once patients were in their 90s.

Finally, Margaret McCartney bids farewell to readers (doi:10. 1136/bmj.k3745). Her columns have been among our most popular content. She closes with 36 pithy observations that summarise the lessons from her four and a half year tenure. Yet again, certainty is elusive. "Medicine is a tough, unglamorous, difficult job" that "often feels impossible to do well ...

Medicine is an absolutely brilliant job." Of two things, though, she is positive: "Being a columnist has been great fun," and her editors are "patient, kind, and clever." Who could doubt that? 